\title{
Current Progress and Future Perspectives of Immune Checkpoint Inhibitors in Biliary Tract Cancer
}

This article was published in the following Dove Press journal: OncoTargets and Therapy

\author{
Poshita-Kumari Seesaha $\mathbb{D}^{1, *}$ \\ Kang-Xin Wang iD ${ }^{2, *}$ \\ Guo-Qun Wang ${ }^{2}$ \\ Ting-Yun Cui ${ }^{2}$ \\ Feng-Jiao Zhao' \\ Lan-Lan Pan (D) ' \\ Xiang-Cheng $\mathrm{Li}^{3}$ \\ Yong-Qian Shu' \\ Xiao-Feng Chen ${ }^{1,2}$ \\ 'Department of Oncology, The First \\ Affiliated Hospital with Nanjing Medical \\ University, Nanjing, 210029, People's \\ Republic of China; ${ }^{2}$ Department of \\ Oncology, Pukou Branch Hospital of \\ Jiangsu Province Hospital (Nanjing Pukou \\ Central Hospital), Nanjing, 21 1800, \\ People's Republic of China; \\ ${ }^{3}$ Hepatobiliary Center of the First \\ Affiliated Hospital with Nanjing Medical \\ University, Nanjing, 210029, People's \\ Republic of China
}

*These authors contributed equally to this work

Correspondence: Xiao-Feng Chen Department of Oncology, The First Affiliated Hospital with Nanjing Medical University, No. 300 Guangzhou Road, Nanjing, 210029, People's Republic of China

Email chenxiaofengnjmu@I63.com

\begin{abstract}
Biliary tract cancer (BTC) is an uncommon and aggressive neoplasm, with most patients presenting in an advanced stage. Systemic chemotherapy is the limited treatment available but is unsatisfactory, while targeted therapy is still awaiting validation from clinical trials. Given the potential effect of immune checkpoint inhibitors (ICIs) in the treatment of BTC, this review aims to summarize the evidence-based benefits and predictive biomarkers for using inhibitors of cytotoxic T-lymphocyte-associated protein-4 (CTLA-4) ligand, or programmed cell death protein-1 and its ligand (PD-1 and PD-L1) as monotherapy or combined with other anti-tumor therapies, while also pointing out certain pitfalls with the use of ICIs which need to be addressed.
\end{abstract}

Keywords: biliary tract cancer, immunotherapy, immune checkpoint inhibitors, clinical evidence, predictive biomarkers

\section{Introduction}

Biliary tract cancer (BTC), though a rare tumor accounting for only $3 \%$ of all gastrointestinal malignancies, is an aggressive disease with increasing global incidence and mortality rate. On account of the unknown etiology, risk factors such as liver fluke infestation, chronic biliary and liver diseases, and metabolic syndrome may be involved in the tumorigenesis and development of the disease. ${ }^{1}$ BTC is composed of tumors that arise from the epithelial cells of the biliary tree, including the subtypes gallbladder cancer (GBC), ampulla of Vater cancer (AVC), intrahepatic cholangiocarcinomas (ICC), and extrahepatic cholangiocarcinomas (ECC). Owing to the particular anatomical structure and malignant biological properties of BTC, recurrent lesions are generally multiple and diffuse, which is an indicator of poor prognosis. Hence, most patients present with advanced BTC when first diagnosed and only $10-45 \%$ can be surgically resected at that time. However, even with radical resection the incidence of tumor recurrence or distant metastasis remains high.

Standard first-line treatment using cisplatin and gemcitabine was reported to have a limited objective response rate (ORR) of $26 \%$ and no more than 1 year overall survival (OS) according to the ABC-02 trial, and there is no alternative systemic chemotherapy for advanced BTC. In the area of targeted agents, clinical trials have historically demonstrated only modest response in BTC patients, although there has been recent improvement in the exploration of novel targets, including recurrent 
fusion events (FGFR2 and ROS1 fusions), somatic mutations in metabolic enzymes (IDHI and 2), and chromatinremodeling genes (ARIDIA, BAP1, PBRM1). ${ }^{2}$ However, owing to the inadequate clinical evidence on the use of novel targeted therapy in BTC patients, more responsive and durable anticancer regimens are required.

Following the approved use of immune checkpoint inhibitors (ICIs) in cancers such as metastatic melanoma and non-small cell lung cancer, there has been growing interest in their use in BTC. ICIs release the immune barrier by blocking cytotoxic T-lymphocyte-associated protein-4 (CTLA-4) ligand, programmed cell death protein-1 and its ligand (PD-1 and PD-L1), and other immune checkpoint molecules, thereby allowing effector $\mathrm{CD} 8^{+}$ $\mathrm{T}$ cells to set up normal antitumor responses which enhance the efficacy of immunotherapy and may offer hope for further advancing treatment options. ${ }^{3}$ The purpose of this review is to delineate the evidence-based potential benefits of ICIs in BTC, either as monotherapy or combined with other rational therapies, while also pointing out certain pitfalls associated with their use.

\section{Role of Immune Checkpoint Inhibitors in BTC}

Most BTC tumors were found to express immune checkpoint molecules (PD-1/PD-L1, CTLA-4, LAG3, IDO1, HAVCR2, BTLA, TNFRSF4, and TNFRSF9) and a decrease in $\mathrm{CD} 8^{+} \mathrm{T}$-cell count within the tumor microenvironment (TME). ${ }^{4,5}$ It was also demonstrated that ex vivo inhibition of these checkpoint molecules led to an increased proliferation of cytotoxic T cells. ${ }^{6}$ By analyzing the relationship between TME and prognosis in BTC, hypermutated tumors and elevated gene expression of immune checkpoint molecules including CTLA-4 and PDL1 occurred with the worst outcome. ${ }^{7-13}$ All of these studies suggested that the role of immune checkpoint molecules expressed in BTC was to actively suppress the host immune response, and thus they were deemed potential targets for future therapy.

However, there is one point to note, which is that immunotherapy itself has so far been inactive in patients with anatomically and molecularly uncharacterized BTC. Hence, more evidence-based learning about genetic instability interacting with susceptibility to ICIs should be offered and explored. First, a study showed that defects in human leukocyte antigen (HLA) class 1 molecule were present in $50 \%$ of ECCs in Chinese patients and might play a role in immune escape by tumor cells. ${ }^{14,15}$ Second, chronic inflammation resulting from the risk factors for BTC (mentioned in the Introduction) leads to oxidative stress and an imbalance between antioxidant enzymes and DNA repair enzymes. Genome-protecting mechanisms are activated subsequently, which can lead to microsatellite instability (MSI) with carcinogenic potential. ${ }^{16}$ The prognostic role of MSI is conflicting among different subtypes of BTC; one study found that high-level microsatellite instability (MSI-H) or deficient mismatch repair (dMMR) was correlated with poor prognosis, while others reported favorable clinical outcomes. Part of the reason behind this contradictory phenomenon is due to the lack of adequate clinical evidence about MSI, which is a rare finding accounting for $2-10 \%$ of BTC cases. ${ }^{17-19}$ Nevertheless, MSI-H or dMMR has consistently been shown to be the best predictor of response toward ICIs. ${ }^{20}$ Besides, the natural human defense mechanisms against tumors can be exploited for anticancer therapy. A report on three cases of tumor regression in hepatitis $\mathrm{C}$ virus (HCV) cirrhosis patients showed a possible role of the host immune system in the defense against hepatobiliary tumors. ${ }^{21}$ Considering all these characteristics of BTC, changes in genomics and host immune responses may facilitate precise treatment with ICIs.

\section{Clinical Evidence of Use of Immune Checkpoint Inhibitors in BTC}

Current findings on the clinical use of immunotherapy in BTC show only limited efficacy of monotherapy ICIs, except in a few patients with specific biomarkers who had responsive disease. In order to increase the efficacy of immunotherapy in BTC, different therapeutic combinations are currently being tested. Infiltration of lymphocytes into the TME has been found to be related to the response rate of PD-1/PD-L1 inhibitors, which provide an opportunity to target the interaction with the microenvironment. Hence, the development of measures for combination may influence the TME and activate the antitumor immune response by different mechanisms. We expand on this clinical evidence in detail in the following sections to show the potential for ICI therapy in BTC.

\section{Monotherapy Immune Checkpoint Inhibitors in Refractory BTC Pembrolizumab}

Pembrolizumab is a humanized monoclonal anti-PD-1 antibody which is currently being evaluated in clinical trials for 
BTC. In the basket KEYNOTE-158 trial, 104 patients with advanced BTC were recruited and treated with pembrolizumab, all of whom had proficient MMR and 61 of whom had PD-L1-positive tumors. Differently from PD-L1 status, the KEYNOTE-028 trial required PD-L1 positivity for all subjects. Results from the KEYNOTE-028 (NCT02054806) and KEYNOTE-158 (NCT02628067) trials involving cohorts with advanced BTC are already available and are summarized in Table 1; the conclusion was drawn that the ORR of pembrolizumab could be improved in PD-L1positive patients, yet with no benefit on the prognosis. ${ }^{22}$ These unsatisfactory results have set the stage for clinical trials aimed at molecularly characterized patients using pembrolizumab in combination therapies for further improving the response rate of ICIs.

\section{Nivolumab}

Nivolumab is a human immunoglobulin $G_{4}$ anti-PD-1 monoclonal antibody. ${ }^{23}$ A clinical trial (NCT02829918) which studied the use of nivolumab in patients with advanced refractory BTC gave some encouraging results. Out of 45 patients who were evaluated with a median follow-up of 13.3 months, 10 showed a partial response (22.0\%). The median progression-free survival (PFS) and OS were 4.0 months (95\% CI: 2.33-5.98) and 14.2 months (95\% CI: 6.64-NA), respectively. The most common side effect was raised alkaline phosphatase, and no other severe adverse events occurred. Hence, nivolumab has been presumed to have an encouraging clinical efficacy with tolerable side effects in patients with advanced refractory BTC. $^{24}$

\section{Durvalumab}

Durvalumab is a PD-L1 inhibitor which has shown acceptable safety profiles but disappointing results in advanced BTC. One study involving 22 Japanese patients with advanced solid tumors, including BTC, used durvalumab in a dose range of $1-20 \mathrm{mg} / \mathrm{kg}$ and for a median duration of 17.1 weeks. The outcome revealed that the ORR was only $4.5 \%$; the response using durvalumab in the treatment of refractory BTC seemed to progressively decline, with a disease control rate (DCR) of $64.0 \%, 36.0 \%$, and $23.0 \%$ at 6,12 , and 24 weeks, respectively. The most common treatment-related adverse effect (TRAE) encountered was rash. ${ }^{25}$ Another study further reported on two cohorts of BTC patients, one receiving durvalumab (D) as a single agent and another receiving combination therapy $(\mathrm{D}+\mathrm{T})$ with tremelimumab, an anti-CTLA-4 antibody. The DCR reached $16.7 \%$ and $32.2 \%$, and the median OS was 8.1 months (95\% CI: 5.6-$10.1)$ and 10.1 months (95\% CI: 6.2-11.4) in the D and D+T cohorts, respectively. Only one death occurred in the $\mathrm{D}+\mathrm{T}$ cohort. ${ }^{26}$ Hence, durvalumab also shows potential efficacy, especially in combination therapies.

\section{M7824}

Based on preclinical data that tumors with poor response toward ICIs have upregulated transforming growth factor$\beta$ (TGF- $\beta$ ) signaling, M7824, as a bifunctional protein consisting of human anti-PD-L1 antibody fused with

Table I Summary of KEYNOTE-I58 and KEYNOTE-28 Trials

\begin{tabular}{|c|c|c|}
\hline Trial & KEYNOTE-I 58 & KEYNOTE-28 \\
\hline Number of patients & 104 & 24 \\
\hline Main inclusion criteria & $\begin{array}{l}\text { Age } \geq 18 \text { years, prior } \geq \text { first-line systemic therapy, no } \\
\text { prior } I C I \text { treatment, ECOG } 0-\mid\end{array}$ & $\begin{array}{l}\text { Age } \geq 18 \text { years, prior } \geq \text { first-line systemic therapy, no } \\
\text { prior } I C I \text { treatment, ECOG } 0-I\end{array}$ \\
\hline Dose of pembrolizumab & $200 \mathrm{mg} \mathrm{q3w}$ & $10 \mathrm{mg} / \mathrm{kg} \mathrm{q} 2 \mathrm{w}$ \\
\hline PD-LI status & $58.7 \%$ were PD-LI positive & All subjects were PD-LI positive \\
\hline Primary endpoint & ORR & ORR \\
\hline Median follow-up (months) & 7.5 & 6.5 \\
\hline ORR & $5.8 \%$ (95\% Cl: $2.1-12.1)$ & I3.0\% (95\% Cl: $2.8-33.6)$ \\
\hline Median OS (months) & 7.4 (95\% Cl: 5.5-9.6) & 6.2 (95\% Cl: 3.8-10.3) \\
\hline PFS (months) & $2.0(95 \% \mathrm{Cl}:$ I.9-2.I) & 1.8 (95\% Cl: I.4-3.7) \\
\hline I2-month OS & $32.7 \%$ & $27.6 \%$ \\
\hline $\begin{array}{l}\text { Grade } 3-5 \text { treatment-related } \\
\text { adverse effects }\end{array}$ & $13.5 \%$ & $16.7 \%$ \\
\hline $\begin{array}{l}\text { Immune-mediated side effects/ } \\
\text { infusion reactions }\end{array}$ & $18.3 \%$ & $20.8 \%$ \\
\hline
\end{tabular}

Abbreviations: PD-LI, programmed cell death protein ligand-I; ORR, objective response rate; OS, overall survival; PFS, progression-free survival; ICI, immune checkpoint inhibitor; ECOG, Eastern Cooperative Oncology Group. 
TGF- $\beta$ receptor II, has a dual function in blocking the immunosuppression signal transduction pathway. Its efficacy has been investigated in a phase I trial with 30 pretreated Asian BTC patients, in which 30 patients received M7824 for a median of 8.9 weeks and five patients were still on active treatment. An ORR was observed in seven patients $(23.3 \%)$, including responses with PD-L1 status (ORRs: PD-L1 positive 25.0\%, PD-L1 negative $15.4 \%$ ). TRAEs occurred in $60 \%$ of patients; the most common were maculopapular rash and pyrexia, as well as increased lipase and rash. Ten patients $(33.3 \%)$ experienced grade 3-5 TRAEs, including septic shock and interstitial lung disease. Hence, the use of M7824 shows promise, in that it has an acceptable safety profile and promising efficacy, with durable responses in $8 / 30$ patients (27\%) across BTC subtypes. ${ }^{27}$

\section{Immune Checkpoint Inhibitors Combined with Systemic Therapies}

Current clinical trials are mainly focusing on the use of ICIs in combination with other systemic therapies in advanced BTC, including chemotherapy, anti-angiogenic drugs, immunotherapy, and other target inhibitors. Chemotherapy (including gemcitabine and 5-FU) can enhance the antigenicity and immunogenicity of tumors by promoting adaptive immune responses. ${ }^{28,29}$ Our center has evaluated the efficacy of SHR-1210 (camrelizumab), an anti-PD-1 antibody, combined with gemcitabine and oxaliplatin (GEMOX) as first-line treatment in advanced BTC in a single-arm exploratory study (NCT03486678). Among 36 evaluable patients, 19 patients had a partial response (PR, 52.8\%), 14 stable disease (SD, 38.9\%), and three progressive disease (PD, 8.3\%) at best. The median PFS and OS were 6.2 months and 12.1 months, respectively. By extensive biomarker analysis, frequent mutations were found in DNA damage repair, cell cycle regulation, and genome instability genes (eg, ARIDIA, STK11, BRCA2, CTNNB1, and MSH2). Tissue analysis showed that patients with $S T K 11(P=0.0254), C T N N B 1$ $(P<0.001)$, and SMARCA4 $(P=0.0181)$ wild type showed significantly longer PFS than those with mutations. Patients with ARIDIA gene wild type showed a tendency for longer PFS $(P=0.0634)$ and significantly longer OS $(P=0.0149)$. Importantly, PD-L1 positivity may be related to longer PFS (tumor proportion score $>1 \%, P=0.08$; immune proportion score $>1 \%, P=0.05$ ) in this study. ${ }^{30}$, Ueno et al described the efficacy of using nivolumab as monotherapy and combined with cisplatin plus gemcitabine (GemCis) in Japanese patients. The median OS and PFS were longer in the combined therapy cohort compared to the monotherapy cohort (5.2 vs 15.4 months; 1.4 vs 4.2 months). ${ }^{31}$ Severe adverse effects were seen in only one patient in the monotherapy cohort and in 11 in the combined therapy cohort. Apart from using monotherapy ICIs combined with chemotherapy, the tolerability and efficacy of dual ICIs combination have been under research. A phase II study on the use of durvalumab (D) \pm tremelimumab (T) and GemCis in chemo-naive advanced BTC has shown significant and safe results, revealing an ORR of more than $70 \%$ in both the GemCis $+\mathrm{D}$ group and GemCis $+\mathrm{D}+\mathrm{T}$ group, and the $\mathrm{DCR}$ in the GemCis $+\mathrm{D}$ group even reached $100 \%$ without many adverse effects. The baseline tissue tumor mutational burden (TMB) did not correlate with prognosis. Reductions in circulating tumor DNA (ctDNA) variant allele frequency (VAF) were more frequent among responders and significantly correlated with ORR $(P<0.015)$ during early $\mathrm{D}+\mathrm{T}$ cycles. PD-L1 expression after the first GemCis cycle, rather than baseline expression, trended with improved PFS. ${ }^{32}$ Results from another recruiting study (NCT02834013) on dual ICIs combination after multiline therapies for advanced BTC are awaited.

BTC is rather heterogeneous according to the different anatomical and molecular subtypes. Unfortunately, even with corresponding agents, targeted therapy usually results in the emergence of resistant clones over time. ${ }^{33}$ Hence, immunotherapy has been added to targeted molecular agents to determine whether this combined therapy can overcome the resistance. In one study, 14 patients who had received at least two previous treatment regimens for stage IV BTC were treated with the multitargeted tyrosine kinase inhibitor of vascular endothelial growth factor (VEGF) receptor lenvatinib plus pembrolizumab or nivolumab. An encouraging ORR of $21.4 \%$, DCR of $92.9 \%$, and median PFS of 5.9 months (95\% CI: 4.2-6.2) were observed, with an acceptable safety profile. $^{34}$ Another study combined VEGF receptor-2 ramucirumab with pembrolizumab in previously treated BTC patients, and achieved mPFS and mOS of just 1.6 and 6.4 months, respectively. ${ }^{35}$ In view of these conflicting outcomes, more clinical trials to verify this combined regimen are expected to commence in the near future. 


\section{Immune Checkpoint Inhibitors Combined with Local Treatment}

Immunotherapy has yet to find its position in the field of BTC treatment, likely because of the lack of antigenic mutations or the immunosuppression status. Nevertheless, optimism remains that the correct combination or sequence of therapies may hold the key to expanding this therapeutic strategy to the majority of patients who do not have specific biomarkers. ${ }^{36}$ Local radiotherapy (RT) is identified as one of the most significant ways to enhance various components of the antigen processing and presentation pathway. According to the KEYNOTE-001 trial and other clinical evidence, previous RT may prolong PFS and OS in patients receiving immunotherapy which functions by increasing antigen release first and then inducing immune cell infiltration. ${ }^{37,38}$ One study which assessed the effect of combining stereotactic body radiotherapy (SBRT) with ICI in one patient with stage IVA ICC and two patients with recurrent ICC following surgery also showed promising results. All three patients had low TMB, dMMR, and MSI, and two of them showed a partial response while the other showed a complete response. Besides, all three showed an abscopal effect. ${ }^{39}$ In another case report, a patient with metastatic GBC showed good response to combined therapy with nivolumab and radiotherapy. ${ }^{40}$ Although only a few patients were evaluated in these reports, the results with combined therapies showed an encouraging outcome, especially in those patients who were not expected to respond to immunotherapy. Moreover, the efficacy of combining ICIs with local treatment modalities such as transarterial catheter chemoembolization (TACE), radiofrequency ablation (RFA), and cryoablation (CA) is being evaluated. ${ }^{41}$ Multiple studies including ICIs as monotherapy or combined with other anti-tumor therapies are ongoing and are listed in Table 2.

\section{Predictive Biomarkers for Immune Checkpoint Inhibitors in BTC}

It is of great significance to note that even though ICIs have shown their merits in the treatment of several cancers, less than $20-30 \%$ of all patients seem to respond to ICIs, ${ }^{42}$ implying that there are underlying factors that influence treatment efficacy with these agents. This has led to the search for predictive biomarkers to guide therapy with ICIs, making its use more selective for maximum benefit. Increased expression of PD-L1 on host cells is a target for ICIs, especially for PD-1/PD-L1 blockademediated tumor regression. ${ }^{43}$ Mody et al assessed PD-L1 expression in a cohort of 652 BTC patients and found that $8.6 \%$ were PD-L1 positive, with the following distribution: GBC $12.3 \%$ (25/203), ICC $7.3 \%$ (27/372), and ECC $5.2 \%(4 / 77) .{ }^{44}$ In the KEYNOTE- 28 and KEYNOTE-158 trials, the objective response with pembrolizumab was $13 \%$ and $5.8 \%$, respectively. Although the two trials included very similar populations, there was a small difference in that only $58.7 \%$ were PD-L1 positive in KEYNOTE-158 cohort, while $100 \%$ were PD-L1 positive in the KEYNOTE-28 cohort, which could partly explain the different clinical outcome and prognosis. Furthermore, in our study using camrelizumab and GEMOX as first-line treatment for BTC, the PD-L1-positive patients had a better response rate of $80 \%$ and a longer PFS than PDL1-negative patients, as mentioned in the section 'Immune Checkpoint Inhibitors Combined with Systemic Therapies', above. ${ }^{30}$

Along with expression of PD-L1 in BTC, two wellknown molecular subsets of cancers, TMB and dMMR/ MSI-H, may also be valuable biomarkers. A study involving 7757 tumor samples from 26 different cancer types, including ECC, showed that TMB exceeded the threshold of 192 non-synonymous mutations (NSMs), mostly in melanoma and lung cancer patients, and these patients had better survival benefit with ICIs. ${ }^{45}$ Sui et al presented two case reports to uphold the importance of NSMs in predicting the response to ICIs. Both were males diagnosed with stage IIIb ICC, PD-L1 expression $<5 \%$, tumorinfiltrating lymphocyte (TIL) frequency $<10 \%$, and MSI $<0.01 \%$, but with marked NSMs, including insertiondeletion (indel) mutations. Both were started on pembrolizumab and tegafur after recurrence of the tumor and both achieved tumor regression. ${ }^{43}$ Another major molecular pathway of genetic instability in cancer is DNA mismatch repair deficiency. The KEYNOTE-158 trial demonstrated that BTC with MSI-H or dMMR showed better response with pembrolizumab. ${ }^{46}$ MSI status may not need further testing for PD-L1 expression or TMB to decide upon ICI therapy. ${ }^{47}$ However, predictive biomarkers may vary from one type to another in BTC, in that PD-L1 expression, MSI, and TMB are more frequent in ICC and GBC than in $\mathrm{ECC},{ }^{48}$ which reflects the heterogeneity and complexity of BTC itself.

Apart from the aforementioned biomarkers, there may be other factors determining treatment efficacy with ICIs since a positive response has been observed in the absence of PD- 
Table 2 Ongoing Trials Using Monotherapy ICls or Combined with Other Anti-Tumor Therapies in BTC

\begin{tabular}{|c|c|c|c|c|c|}
\hline Regimen & Target(s) & Phase & Status & $\begin{array}{l}\text { No. of } \\
\text { Partcipants }\end{array}$ & $\begin{array}{l}\text { Clinical Trial } \\
\text { Identifier }\end{array}$ \\
\hline \multicolumn{6}{|l|}{ Single-agent ICls } \\
\hline Toripalimab (JSOOI) & PD-I & $\mathrm{lb} / \mathrm{II}$ & Not yet recruiting & 20 & NCT03867370 \\
\hline M7824 & PD-LI & ॥ & Recruiting & $14 \mid$ & NCT0383366I \\
\hline STI-303I & PD-LI & ॥ & Not yet recruiting & 220 & NCT03999658 \\
\hline Pembrolizumab & PD-I & II & Recruiting & 1350 & NCT02628067 \\
\hline \multicolumn{6}{|l|}{$\mathrm{ICls}+\mathrm{ICls}$} \\
\hline Pembrolizumab $+X_{m A b^{\circledR}} 2284 I$ & PD-I, CTLA-4 & 1 & Recruiting & 242 & NCT03849469 \\
\hline Nivolumab + ipilimumab & PD-I, CTLA-4 & II & Recruiting & 707 & NCT028340I3 \\
\hline \multicolumn{6}{|l|}{ ICls + chemotherapy } \\
\hline SHR-I210 (camrelizumab) + GEMOX & PD-I & $\|$ & Awaiting final results & 38 & NCT03486678 \\
\hline Pembrolizumab + capecitabine-oxaliplatin & PD-I & II & Recruiting & 19 & NCT03IIII32 \\
\hline Pembrolizumab + gemcitabine-cisplatin & PD-I & III & Recruiting & 788 & NCT04003636 \\
\hline M7824 + gemcitabine-cisplatin & PD-LI & $\|/\| I I$ & Recruiting & 524 & NCT03267940 \\
\hline Durvalumab + guadecitabine & PD-LI & $\mathrm{lb}$ & Recruiting & 90 & NCT0325776I \\
\hline Durvalumab + gemcitabine/cisplatin & PD-LI & 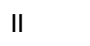 & Recruiting & 474 & NCT03875235 \\
\hline $\begin{array}{l}\text { Durvalumab, tremelimumab + gemcitabine/ } \\
\text { gemcitabine-cisplatin }\end{array}$ & PD-LI,CTLA-4 & ॥ & Recruiting & 128 & NCT03473574 \\
\hline Durvalumab, tremelimumab + gemcitabine/cisplatin & PD-LI,CTLA-4 & ॥ & Recruiting & 31 & NCT03046862 \\
\hline \multicolumn{6}{|l|}{$\mathrm{ICls}+$ protein kinase inhibitors } \\
\hline Pembrolizumab + lenvatinib & PD-I, VEGFR & llb & Recruiting & 50 & NCT03895970 \\
\hline Pembrolizumab + pemigatinib & PD-I, FGFR & $1 / I I$ & Recruiting & 325 & NCT02393248 \\
\hline TQB2450 + anlotinib & PD-LI, VEGFR & $1 / I I$ & Recruiting & 42 & NCT03996408 \\
\hline Atezolizumab + cobimetinib & PD-LI, MEK & II & Active, not recruiting & 82 & NCT0320I458 \\
\hline \multicolumn{6}{|l|}{ ICls + radiotherapy } \\
\hline Durvalumab, tremelimumab + RT & PD-LI, CTLA-4 & $\|$ & Recruiting & 70 & NCT03482102 \\
\hline \multicolumn{6}{|l|}{$\mathrm{ICls}+$ other therapies } \\
\hline JSOOI + GEMOX + lenvatinib & PD-I, VEGFR & II & Recruiting & 30 & NCT0395I597 \\
\hline Nivolumab + entinostat & PD-I, HDAC & II & Recruiting & 54 & NCT03250273 \\
\hline Nivolumab + TPST-II 20 & PD-I, PPAR $\alpha$ & $\mathrm{l} / \mathrm{lb}$ & Recruiting & 338 & NCT03829436 \\
\hline Pembrolizumab + CD8 T cells & PD-I & 1 & Enrolling by invitation & 40 & NCT02757395 \\
\hline Pembrolizumab + allogenic NK cells & PD-I & l/lla & Not yet recruiting & 40 & NCT03937895 \\
\hline Atezolizumab + PEG-PH20 & PD-LI & $\mathrm{lb}$ & Recruiting & 85 & NCT03267940 \\
\hline Avelumab + nedisertib & PD-LI & $1 / I 1$ & Not yet recruiting & 92 & NCT04068194 \\
\hline
\end{tabular}

L1/PD-1 expression, increased TMB, and MSI. ${ }^{49}$ For instance, $S T K-11$ was reported to be a negative biomarker in NSCLC patients treated with ICIs. In our study focused on biomarker exploration for SHR-1210 plus GEMOX therapy in BTC, there were two patients with STK-11 mutation, both of whom showed progressive disease, indicating that STK-11 mutation may also be a negative biomarker in BTC patients treated with ICIs. ${ }^{50}$ Besides, HHLA2, identified as a novel T-cell immune checkpoint molecule, was found to be more frequently expressed than PD-L1/PD-1 in ICC, and may be a novel target for immunotherapy. ${ }^{51}$

\section{Future Perspectives for Immune Checkpoint Inhibitors in BTC}

Current progress with ongoing research and future perspectives for ICIs in BTC seems to be both promising and challenging. Most BTC tumors are thought to arise from a chronic inflammatory status in the biliary tract 
accompanied by overexpression of immune checkpoint molecules. Thus, the immune system plays an essential role in the etiology of BTC and it is a particular TME that provides the rationale for ICI therapy in BTC. However, the results obtained are yet to match those of ICI monotherapy in hepatocellular cancer and non-small cell lung cancer. ${ }^{52}$ It is unclear whether ICI therapy could surpass or supplement molecular targeted therapy in BTC. With more extensive research, combined therapy has proven to be more efficient than monotherapy, and seems to be the future direction for this form of treatment. ${ }^{28-32,34,36-41}$ Nevertheless, the ideal combination partner, whether chemotherapy, molecular targeted therapy, or radiotherapy, needs to be evaluated, and which one is the best is still a matter of ongoing research. The proper dose, duration, and the sequence should be evaluated to obtain the maximum clinical benefits with limited toxicity.

It is also worth noting that the most challenging part in the use of ICIs is finding predictive biomarkers to determine the population that will benefit. One reason for this is the heterogeneity and complexity of BTC itself. On the other hand, it could also be due to the lack of clinical evidence on the relationship between predictive biomarkers and prognosis. For the currently known biomarkers such as PD-L1/PD-1 expression, increased TMB and MSI, that are used to predict prognosis, evaluate the therapeutic response, and provide selection tools for BTC treatment, information on their application in clinical practice is still unclear, including whether they should be used singly or as part of a package, the optimum time for detection, and whether more credible biomarkers may exist. As well as predictive biomarkers, other factors also affect the response to ICIs. For instance, the gut microbiome has been much studied in conjunction with colorectal and hepatobiliary-pancreatic carcinomas. Gut-specific microbes, such as Bacteroides fragilis and Bifidobacterium, have an effect on the therapeutic response to ICIs. Therefore, the gut flora can be manipulated with probiotics to enhance the response to ICIs, and can even be used as potential biomarkers. ${ }^{53}$ Elevated C-reactive protein (CRP) has been shown to affect the efficacy of ICIs in Chinese patients with advanced melanoma. ${ }^{54}$ A systemic review and meta-analysis proposed the possible role of gender, with females being better candidates for ICI therapy, ${ }^{55}$ but this was refuted by two other studies which found no significant relationship between gender and response. ${ }^{56,57}$ Another study associated age with therapeutic outcome; a survival benefit in metastatic melanoma patients who received ICIs was seen in both young and older ( $>60$ years) age groups, but the result was more prominent in older age groups. ${ }^{58}$

With increased characterization of the molecular and biological landscape, more and more potential immunerelated biomarkers or biological information will be used to identify vulnerabilities that can be exploited in clinical trials to provide the key to their successful application in the clinical setting. The hope is that ongoing and future clinical trials will eventually solve all the related concerns about ICIs in BTC. Moreover, since combined therapy is superior to single-agent therapy, active research avenues exploring the development of novel therapeutics that target the crosstalk between cancer and TME, the cellular pathways of cell death to improve life, mediators of chemoresistance, and potential RNA therapeutics should follow the development of ICI therapy. ${ }^{59}$

\section{Abbreviations}

AVC, ampulla of Vater cancer; BTC, biliary tract cancer; CA, cryoablation; ctDNA, circulating tumor DNA; CRP, C-reactive protein; CTLA-4, cytotoxic T-lymphocyteassociated protein-4; DCR, disease control rate; dMMR, deficient mismatch repair; ECC, extrahepatic cholangiocarcinoma; GBC, gallbladder cancer; $\mathrm{HCV}$, hepatitis $\mathrm{C}$ virus; HLA, human leukocyte antigen; ICC, intrahepatic cholangiocarcinoma; ICI, immune checkpoint inhibitor; MSI, microsatellite instability; MSI-H, high-level microsatellite instability; NSM, non-synonymous mutation; ORR, objective response rate; OS, overall survival; PD-1, programmed cell death protein-1; PD-L1, programmed cell death protein ligand-1; PFS, progression-free survival; RFA, radiofrequency ablation; RT, radiotherapy; SBRT, stereotactic body radiotherapy; TACE, transarterial catheter chemoembolization; TGF- $\beta$, transforming growth factor- $\beta$; TIL, tumorinfiltrating lymphocyte; TMB, tumor mutational burden; TME, tumor microenvironment; TRAE, treatment-related adverse effect; VAF, variant allele frequency; VEGF, vascular endothelial growth factor.

\section{Author Contributions}

All authors made substantial contributions to conception and design, acquisition of data, or analysis and interpretation of data; took part in drafting the article or revising it critically for important intellectual content; agreed to submit to the current journal; gave final approval of the 
version to be published; and agree to be accountable for all aspects of the work. Poshita-Kumari Seesaha and KangXin Wang are co-first authors and contributed equally to this work.

\section{Funding}

The study was financially supported by Jiangsu Province 333 High-Level Talents Project, innovation funds from the Chinese Society of Clinical Oncology Youth Committee, the Advanced Health Talent of Six-One Project of Jiangsu Province, and a joint research project by Southeast University and Nanjing Medical University.

\section{Disclosure}

The authors declare that they have no conflicts of interest for this work.

\section{References}

1. Tariq N-U-A, McNamara MG, Valle JW. Biliary tract cancers: current knowledge, clinical candidates and future challenges. Cancer Manag Res. 2019;11:2623-2642. doi:10.2147/CMAR.S157092

2. Adeva J, Sangro B, Salati M, et al. Medical treatment for cholangiocarcinoma. Liver Int. 2019;39(S1):123-142. doi:10.1111/ liv. 14100

3. Abril-Rodriguez G, Ribas A. SnapShot: immune Checkpoint Inhibitors. Cancer Cell. 2017;31(6):848-e1. doi:10.1016/j.ccell.20 17.05.010

4. Miura T, Yoshizawa T, Hirai H, et al. Prognostic impact of CD163+ macrophages in tumor stroma and CD8+ T-cells in cancer cell nests in invasive extrahepatic bile duct cancer. Anticancer Res. 2017;37 (1):183-190. doi:10.21873/anticanres.11304

5. Nakamura H, Arai Y, Totoki Y, et al. Genomic spectra of biliary tract cancer. Nat Genet. 2015;47(9):1003-1010. doi:10.1038/ng.3375

6. Zhou G, Sprengers D, Mancham S, et al. Reduction of immunosuppressive tumor microenvironment in cholangiocarcinoma by ex vivo targeting immune checkpoint molecules. J Hepatol. 2019;71 (4):753-762. doi:10.1016/j.jhep.2019.05.026

7. Kim MH, Jang M, Kim H, Lee WJ, Kang CM, Choi HJ. Distinct immunological properties of the two histological subtypes of adenocarcinoma of the ampulla of vater. Cancer Immunol Immunother. 2019;68(3):443-454. doi:10.1007/s00262-018-02293-6

8. Kriegsmann M, Roessler S, Kriegsmann K, et al. Programmed cell death ligand 1 (PD-L1, CD274) in cholangiocarcinoma - correlation with clinicopathological data and comparison of antibodies. $B M C$ Cancer. 2019;19(1):72. doi:10.1186/s12885-018-5254-0

9. Ueno T, Tsuchikawa T, Hatanaka KC, et al. Prognostic impact of programmed cell death ligand 1 (PD-L1) expression and its association with epithelial-mesenchymal transition in extrahepatic cholangiocarcinoma. Oncotarget. 2018;9(28):20034-20047. doi:10. 18632/oncotarget. 25050

10. Fontugne J, Augustin J, Pujals A, et al. PD-L1 expression in perihilar and intrahepatic cholangiocarcinoma. Oncotarget. 2017;8(15): 24644-24651. doi:10.18632/oncotarget.15602

11. Ha H, Nam AR, Bang JH, et al. Soluble programmed death-ligand 1 (sPDL1) and neutrophil-to-lymphocyte ratio (NLR) predicts survival in advanced biliary tract cancer patients treated with palliative chemotherapy. Oncotarget. 2016;7(47):76604-76612. doi:10.18632/ oncotarget. 12810
12. Ahn S, Lee Y, Kim JW, et al. Programmed cell death ligand-1 (PD-L1) expression in extrahepatic biliary tract cancers: a comparative study using 22C3, SP263 and E1L3N anti-PD-L1 antibodies. Histopathology. 2019;75(4):526-536. doi:10.1111/his.13 901

13. Sangkhamanon S, Jongpairat $\mathrm{P}$, Sookprasert A, et al. Programmed death-ligand 1 (PD-L1) expression associated with a high neutrophil/ lymphocyte ratio in cholangiocarcinoma. Asian Pac J Cancer Prev. 2017;18(6):1671-1674. doi:10.22034/APJCP.2017.18.6.1671

14. Yu F, Gong L, Mo Z, et al. Programmed death ligand-1, tumor infiltrating lymphocytes and HLA expression in Chinese extrahepatic cholangiocarcinoma patients: possible immunotherapy implications. Biosci Trends. 2019;13(1):58-69. doi:10.5582/bst.2019.01003

15. Sabbatino F, Villani V, Yearley JH, et al. PD-L1 and HLA class I antigen expression and clinical course of the disease in intrahepatic cholangiocarcinoma. Clin Cancer Res. 2016;22(2):470-478. doi:10. 1158/1078-0432.CCR-15-0715

16. Loilome W, Kadsanit S, Muisook K, et al. Imbalanced adaptive responses associated with microsatellite instability in cholangiocarcinoma. Oncol Lett. 2017;13(2):639-646. doi:10.3892/ ol.2016.5477

17. Goeppert B, Roessler S, Renner M, et al. Low frequency of mismatch repair deficiency in gallbladder cancer. Diagn Pathol. 2019;14(1):36. doi:10.1186/s13000-019-0813-5

18. Goeppert B, Roessler S, Renner M, et al. Mismatch repair deficiency is a rare but putative therapeutically relevant finding in non-liver fluke associated cholangiocarcinoma. Br J Cancer. 2019;120 (1):109-114. doi:10.1038/s41416-018-0199-2

19. Winkelmann R, Schneider M, Hartmann S, et al. Microsatellite instability occurs rarely in patients with cholangiocarcinoma: a retrospective study from a German Tertiary Care Hospital. Int J Mol Sci. 2018;19(5):1421. doi:10.3390/ijms19051421

20. Czink E, Kloor M, Goeppert B, et al. Successful immune checkpoint blockade in a patient with advanced stage microsatellite-unstable biliary tract cancer. Cold Spring Harb Mol Case Stud. 2017;3(5): a001974. doi:10.1101/mcs.a001974

21. Parks AL, McWhirter RM, Evason K, Kelley RK. Cases of spontaneous tumor regression in hepatobiliary cancers: implications for immunotherapy? J Gastrointest Cancer. 2015;46(2):161-165. doi:10.1007/s12029-015-9690-7

22. Bang Y-J, Ueno M, Malka D, et al. Pembrolizumab (pembro) for advanced biliary adenocarcinoma: results from the KEYNOTE-028 (KN028) and KEYNOTE-158 (KN158) basket studies. J Clin Oncol. 2019;37(15_suppl):4079. doi:10.1200/JCO.2019.37.15_suppl.4079

23. Finkelmeier F, Waidmann O, Trojan J. Nivolumab for the treatment of hepatocellular carcinoma. Expert Rev Anticancer Ther. 2018;18 (12):1169-1175. doi:10.1080/14737140.2018.1535315

24. Kim RD, Kim DW, Alese OB, et al. A phase II study of nivolumab in patients with advanced refractory biliary tract cancers (BTC). J Clin Oncol. 2019;37(15_suppl):4097. doi:10.1200/JCO.2019.37.15_suppl. 4097

25. Fujiwara Y, Iguchi H, Yamamoto N, et al. Tolerability and efficacy of durvalumab in Japanese patients with advanced solid tumors. Cancer Sci. 2019;110(5):1715-1723. doi:10.1111/cas.14003

26. Ioka T, Ueno M, Oh D-Y, et al. Evaluation of safety and tolerability of durvalumab (D) with or without tremelimumab (T) in patients (pts) with biliary tract cancer (BTC). J Clin Oncol. 2019;37(4_suppl):387. doi:10.1200/JCO.2019.37.4_suppl.387

27. Yoo C, Oh DY, Choi HJ, et al. M7824 (MSB0011359C), a bifunctional fusion protein targeting PD-L1 and TGF- $\beta$, in Asian patients with pretreated biliary tract cancer: preliminary results from a phase I trial. Ann Oncol. 2018;29:viii258-viii9. doi:10.1093/ annonc/mdy 282.140

28. Koido S, Kan S, Yoshida K, et al. Immunogenic modulation of cholangiocarcinoma cells by chemoimmunotherapy. Anticancer Res. 2014;34(11):6353-6361. 
29. Liu WM, Fowler DW, Smith P, et al. Pre-treatment with chemotherapy can enhance the antigenicity and immunogenicity of tumours by promoting adaptive immune responses. Br J Cancer. 2010;102 (1):115-123. doi:10.1038/sj.bjc. 6605465

30. Chen $\mathrm{X}, \mathrm{Wu} \mathrm{X}, \mathrm{Wu} \mathrm{H}$, et al. SHR-1210 plus GEMOX as first line treatment in biliary tract cancer: results from a single-arm exploratory study. J Clin Oncol. 2019;37(15_suppl):4092. doi:10.1200/ JCO.2019.37.15_suppl.4092

31. Ueno M, Ikeda $\bar{M}$, Morizane $\mathrm{C}$, et al. Nivolumab alone or in combination with cisplatin plus gemcitabine in Japanese patients with unresectable or recurrent biliary tract cancer: a non-randomised, multicentre, open-label, Phase 1 study. Lancet Gastroenterol Hepatol. 2019;4(8):611-621. doi:10.1016/S2468-1253(19)30086-X

32. Oh D-Y, Lee K-H, Lee D-W, et al. Phase II study assessing tolerability, efficacy, and biomarkers for durvalumab (D) \pm tremelimumab (T) and gemcitabine/cisplatin (GemCis) in chemo-naïve advanced biliary tract cancer (aBTC). J Clin Oncol. 2020;38(15_suppl):4520. doi:10.1200/JCO.2020.38.15_suppl.4520

33. Rizvi S, Gores GJ. Emerging molecular therapeutic targets for cholangiocarcinoma[J]. J Hepatol. 2017;67(3):632. doi:10.1016/j. jhep.2017.03.026

34. Lin J, Shi W, Zhao S, et al. Lenvatinib plus checkpoint inhibitors in patients (pts) with advanced intrahepatic cholangiocarcinoma (ICC): preliminary data and correlation with next-generation sequencing $J$ Clin Oncol. 2018;36(4_suppl):500. doi:10.1200/JCO.2018.36. 4 suppl.500

35. Arkenau HT, Martin-Liberal J, Calvo E, et al. Ramucirumab plus pembrolizumab in patients with previously treated advanced or metastatic biliary tract cancer: nonrandomized, open-label, phase I trial (JVDF). Oncologist. 2018;23(12):1407-e136. doi:10.1634/theoncologist.2018-0044

36. Mizrahi JD, Shroff RT. New treatment options for advanced biliary tract cancer. Curr Treat Options Oncol. 2020;21(8). doi:10.1007/ s11864-020-00767-3

37. Chajon E, Castelli J, Marsiglia H, De Crevoisier R. The synergistic effect of radiotherapy and immunotherapy: a promising but not simple partnership. Crit Rev Oncol Hematol. 2017;111:124-132. doi:10.1016/j.critrevonc.2017.01.017

38. Shaverdian N, Lisberg AE, Bornazyan K, et al. Previous radiotherapy and the clinical activity and toxicity of pembrolizumab in the treatment of non-small-cell lung cancer: a secondary analysis of the KEYNOTE-001 phase 1 trial. Lancet Oncol. 2017;18(7):895-903. doi:10.1016/S1470-2045(17)30380-7

39. Liu X, Yao J, Song L, Zhang S, Huang T, Li Y. Local and abscopal responses in advanced intrahepatic cholangiocarcinoma with low TMB, MSS, pMMR and negative PD-L1 expression following combined therapy of SBRT with PD-1 blockade. J Immunother Cancer. 2019;7(1):204. doi:10.1186/s40425-019-0692-z

40. Kong W, Wei J, Liu J, et al. Significant benefit of nivolumab combining radiotherapy in metastatic gallbladder cancer patient with strong PD-L1 expression: a case report. Onco Targets Ther. 2019;12: 5389-5393. doi:10.2147/OTT.S208926

41. Kalbasi A, June CH, Haas N, Vapiwala N. Radiation and immunotherapy: a synergistic combination. J Clin Invest. 2013;123 (7):2756-2763. doi:10.1172/JCI69219

42. Park YJ, Kuen DS, Chung Y. Future prospects of immune checkpoint blockade in cancer: from response prediction to overcoming resistance. Exp Mol Med. 2018;50(8):109. doi:10.1038/s12276-0180130-1

43. Sui M, Li Y, Wang H, et al. Two cases of intrahepatic cholangiocellular carcinoma with high insertion-deletion ratios that achieved a complete response following chemotherapy combined with PD-1 blockade. J Immunother Cancer. 2019;7(1):125. doi:10.1186/s40425019-0596-y
44. Mody K, Starr J, Saul M, et al. Patterns and genomic correlates of PD-L1 expression in patients with biliary tract cancers. J Gastrointest Oncol. 2019;10(6):1099-1109. doi:10.21037/jgo.2019.08.08

45. Colli LM, Machiela MJ, Myers TA, Jessop L, Yu K, Chanock SJ. Burden of nonsynonymous mutations among TCGA cancers and candidate immune checkpoint inhibitor responses. Cancer Res. 2016;76(13):3767-3772. doi:10.1158/0008-5472.CAN-16-0170

46. Marabelle A, Le DT, Ascierto PA, et al. Efficacy of pembrolizumab in patients with noncolorectal high microsatellite instability/mismatch repair-deficient cancer: results from the phase II KEYNOTE-158 study. J Clin Oncol. 2020;38(1):1-10. doi:10.1200/JCO.19.02105

47. Luchini C, Bibeau F, Ligtenberg MJL, et al. ESMO recommendations on microsatellite instability testing for immunotherapy in cancer, and its relationship with PD-1/PD-L1 expression and tumour mutational burden: a systemic review-based approach. Ann Oncol. 2019;30 (8):1232-1243. doi:10.1093/annonc/mdz116

48. Weinberg BA, Xiu J, Lindberg MR, et al. Molecular profiling of biliary cancers reveals distinct molecular alterations and potential therapeutic targets. J Gastrointest Oncol. 2019;10(4):652-662. doi:10.21037/jgo.2018.08.18

49. Salem ME, Puccini A, Grothey A, et al. Landscape of tumor mutation load, mismatch repair deficiency, and PD-L1 expression in a large patient cohort of gastrointestinal cancers. Mol Cancer Res. 2018;16 (5):805-812. doi:10.1158/1541-7786.MCR-17-0735

50. Chen X, Wu H, Wu X, et al. Biomarker exploration for SHR-1210 plus GEMOX as first-line treatment in advanced biliary tract cancer. $J$ Clin Oncol. 2020;38(4_suppl):536. doi:10.1200/JCO.2020.38. 4 suppl.536

51. Jing CY, Fu YP, Yi Y, et al. HHLA2 in intrahepatic cholangiocarcinoma: an immune checkpoint with prognostic significance and wider expression compared with PD-L1. J Immunother Cancer. 2019;7 (1):77. doi:10.1186/s40425-019-0554-8

52. Uemura T, Hida T. Durvalumab showed long and durable effects after chemoradiotherapy in stage III non-small cell lung cancer: results of the PACIFIC study. J Thorac Dis. 2018;10(Suppl S9):S1108-s12. doi:10.21037/jtd.2018.03.180

53. Mima K, Nakagawa S, Sawayama $\mathrm{H}$, et al. The microbiome and hepatobiliary-pancreatic cancers. Cancer Lett. 2017;402:9-15. doi:10.1016/j.canlet.2017.05.001

54. Zhao L, Yang Y, Ma B, et al. Factors influencing the efficacy of anti-PD-1 therapy in Chinese Patients with advanced melanoma. J Oncol. 2019;2019:6454989. doi:10.1155/2019/6454989

55. Conforti F, Pala L, Bagnardi V, et al. Sex-based heterogeneity in response to lung cancer immunotherapy: a systemic review and meta-analysis. $J$ Natl Cancer Inst. 2019;111(8):772-781. doi:10.1093/jnci/djz094

56. Wallis CJD, Butaney M, Satkunasivam R, et al. Association of patient sex with efficacy of immune checkpoint inhibitors and overall survival in advanced cancers: a systemic review and meta-analysis. JAMA Oncol. 2019;5(4):529-536. doi:10.1001/jamaoncol.2018.5904

57. Hassler MR, Abufaraj M, Kimura S, et al. Impact of patients' gender on efficacy of immunotherapy in patients with metastatic kidney cancer: a systemic review and meta-analysis. Clin Genitourin Cancer. 2019.

58. Jain V, Hwang WT, Venigalla S, et al. Association of age with efficacy of immunotherapy in metastatic melanoma. Oncologist. 2019.

59. José J, Marin G, Prete MG, et al. Current and novel therapeutic opportunities for systemic therapy in biliary cancer. $\mathrm{Br} J$ Cancer. 2020 . 


\section{Publish your work in this journal}

OncoTargets and Therapy is an international, peer-reviewed, open access journal focusing on the pathological basis of all cancers, potential targets for therapy and treatment protocols employed to improve the management of cancer patients. The journal also focuses on the impact of management programs and new therapeutic agents and protocols on patient perspectives such as quality of life, adherence and satisfaction. The manuscript management system is completely online and includes a very quick and fair peer-review system, which is all easy to use. Visit http://www.dovepress.com/ testimonials.php to read real quotes from published authors.

Submit your manuscript here: https://www.dovepress.com/oncotargets-and-therapy-journal 